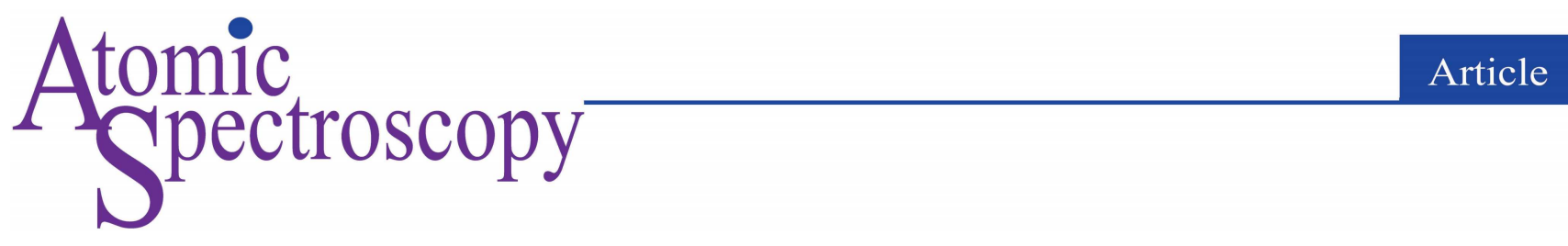

\title{
A Novel Strategy of Anti-interference for Solid Dilution in Elemental Analysis Using Spontaneous Surface Dispersion Theory
}

\author{
Bo Wang, ${ }^{\mathrm{a}, \mathrm{d}, \uparrow}$ Tengpeng Liu, ${ }^{\mathrm{a}, \$}$ Jixin Liu, ${ }^{\mathrm{a}, \mathrm{b}, *}$ Xuefei Mao, ${ }^{\mathrm{a}, *}$ Xing Na, ${ }^{\mathrm{b}}$ Guoying Chen, ${ }^{\mathrm{c}}$ \\ Zhaohui Lv, and Yongzhong Qian ${ }^{\mathrm{a}}$ \\ ${ }^{a}$ Institute of Quality Standard and Testing Technology for Agro-products, Chinese Academy of Agricultural Sciences, and Key Laboratory of Agro-food \\ Safety and Quality, Ministry of Agriculture, Beijing 100081, P.R. China \\ ${ }^{\mathrm{b}}$ Beijing Ability Technique Company, Limited, Beijing 100081, P.R. China \\ ${ }^{c}$ U.S. Department of Agriculture, Agricultural Research Service, Eastern Regional Research Center, 600 E. Mermaid Lane, Wyndmoor, PA 19038, USA \\ d Jilin Province Product Quality Supervision and Inspection Institute, Changchun 130000, P.R. China
}

Received: Jun. 1, 2020; Revised: Jun. 15, 2020; Accepted: Jun. 15, 2020; Published: Jun. 25, 2020.

DOI: 10.46770/AS.2020.03.004

ABSTRACT: A novel idea of anti-interference via solid dilution driven by spontaneous surface dispersion (SSD) is herewith first rendered for elemental analysis to determine trace cadmium in a solid sample, which is similar to the spontaneous monolayer dispersion theory originally applied to heterogeneous catalysts at the percentage level. After pre-ashing with agitation, $\mathrm{Cd}$ in a solid sample can be efficiently and quickly diluted via $\mathrm{SSD}$ on $\alpha-\mathrm{Al}_{2} \mathrm{O}_{3}$ support by heating at $600-700{ }^{\circ} \mathrm{C}$ for $30-40$ min, thus leading to $>180$-fold dilution. Secondary ion mass spectrometry (SIMS) proved that the surface concentration of $\mathrm{Cd}$ on the $\alpha-\mathrm{Al}_{2} \mathrm{O}_{3}$ support significantly increased, indicating that SSD occurred to Cd. The mechanism was thereby confirmed and then deduced. In essence, the proposed SSD is a dilution process for solid samples on the support's surface near Tammann temperature, where homogeneous monolayer or sub-monolayer coverage forms to reduce and even avoid contact of the target elements with interferent. The anti-interference effect has been completely verified via the matrix interference evaluation for $\mathrm{Cd}$ in a rice sample. In addition, SSD solid dilution consumes very little $\alpha-\mathrm{Al}_{2} \mathrm{O}_{3}$ for solid sample analysis after ashing, and shows reliability, precision, simplicity, and is green chemistry. This SSD approach opens a novel horizon for anti-interference research in the elemental field of analytical chemistry.
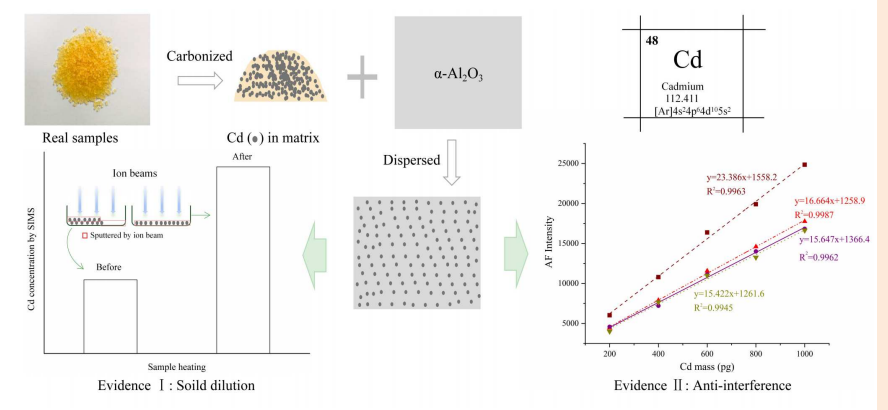

\section{INTRODUCTION}

In analytical instrument design, it is ideal to measure analyte concentrations ranging from the lowest level to $100 \%$, and obtain accurate and precise results without interference. However, in the real world, an analytical instrument is lacking due to multiple factors. For example, L'vov first utilized a graphite crucible in 1957 to fulfill electrothermal vaporization (ETV) of $\mathrm{NaCl}$ for atomic absorption spectrometry (AAS). ${ }^{1}$ In fact, it is the rudiment of modern graphite furnace AAS (GF-AAS) derived from the Massmann furnace design. From then on, due to high sampling efficiency and versatility, ETV has been employed as the solid sampling approach to couple with various atomic spectrometric techniques, such as ETV-AAS, ${ }^{2,3}$ ETV-atomic fluorescence spectrometry (ETV-AFS), ${ }^{4,5}$ ETV-inductively coupled plasma optical emission spectrometry (ICP-OES), ${ }^{6,7}$ and ETV-inductively coupled plasma mass spectrometry (ICP-MS).$^{8-10}$ For real sample analysis, solid sampling became simple and rapid without requiring acid digestion. However, the use of liquid sampling with atomic spectrometric instruments still predominates today. Why does solid sampling analysis not win favor from researchers and 
users in laboratories? To discuss this problem in depth, in this study $\mathrm{Cd}$ was chosen as the analyte considering the contamination severity found today in terms of food safety and quality of electronic products ${ }^{11,12}$ in China. By using a liquid sampling atomic spectrometer, such as GF-AAS to measure $\mathrm{Cd}$, acid digestion removes the organics in the analyte and homogeneously dilutes $\mathrm{Cd}$ in a liquid medium, then chemical modifiers ${ }^{13,14}$ such as $\mathrm{Pd}\left(\mathrm{NO}_{3}\right)_{2}$ and $\left(\mathrm{NH}_{4}\right)_{2} \mathrm{HPO}_{4}$ can further eliminate elemental interference, and finally apply background correction ${ }^{15,16}$ like the Zeeman effect ${ }^{17-19}$ which serves to diminish spectral interference. Among them, the dilution effect caused by the acid digestion process $^{20,21}$ is always ignored, where the interferent concentration would be decreased to weaken the matrix effect to some extent. Moreover, if the dilution ratio is enhanced enough to decrease the interferent below the threshold level, interference-free measurement can be obtained. So, the above-mentioned interference eliminations are all based on the diluted liquid with the analyte and interferent at low levels. Once meeting the interferent with several orders of magnitude higher than before, the existing anti-interference approaches would become invalid, like the problems generally observed with solid sampling analysis.

In recent years, attempts were frequently made to address the limitations derived from matrix effects, such as matrix modifier, ${ }^{22,23}$ standard addition, ${ }^{24}$ matrix matching calibrations, ${ }^{25}$ background correction, ${ }^{15,16,26}$ and so on. But their complete elimination is still not possible. Among them, matrix matching is one of the most effective means, but there is a lack of serial certified reference materials (CRMs) with different concentration levels of the analyte(s). ${ }^{4,5,27}$ To improve matrix matching, analyte fusion with borate or glass, embedding the analyte in a polymer resin, preparation of a pressed disk in the presence of a binder, solid/solution mixture with spiking/drying, etc. ${ }^{27-29}$ was reported. However, these methods are still laborious, tedious, not suitable for other matrices, such as foods, and have unknown homogeneity, which compromises the precision of the analysis. ${ }^{30}$ To the best of our knowledge, dilution is still performed in liquid or gas phases only to warrant analyte homogeneity in the diluted media rather than in the solid phase. Therefore, the accuracy and stability of the solid sampling techniques are always in doubt. ${ }^{31,32}$ If solid phase dilution is achievable with a liquid medium, it might be a novel feasible solution for anti-interference. However, it is unfortunate that quantitative dilution of a solid sample remains a dilemma.

Why is it so difficult to dilute solid phase? As we know, the interparticle force of a solid is much higher than for a liquid, thus its bulk diffusion coefficient is so low that components in a solid cannot be homogenized as a liquid. For this reason, what is usually done is to grind or break the solid sample and medium into micro particles to mix sufficiently with each other. However, single homogenization cannot reach the desired effect. In previous studies on the synthesis and preparation of heterogeneous catalysts, ${ }^{33,34}$ the spontaneous dispersion theory demonstrated an excellent capacity of surface dispersion for components at the percentage level. ${ }^{35,36}$ Though catalyst synthesis seems completely irrelevant with our theme, spontaneous dispersion is essentially a physicochemical phenomenon by which substances tend to evenly disperse on a support surface with increased entropy at an adequate temperature after a sufficient time lapse.

Xie and Tang ${ }^{37,38}$ first applied the spontaneous dispersion theory to elucidate the synthesis process of heterogeneous catalysts. In their study, ${ }^{37,38}$ when solid compounds such as oxides or salts were mixed with a support with a high melting point, such as $\mathrm{SiO}_{2}$ and $\mathrm{Al}_{2} \mathrm{O}_{3}$, the former dispersed spontaneously on the support surface until a thermodynamically stable and homogeneous monolayer or sub-monolayer coverage formed after sufficient heat treatment. ${ }^{39-}$ ${ }^{41}$ In essence, this homogenization is a dilution process of solid compounds on the surface of the supports due to the higher surface diffusion speed at a certain temperature; in addition, the formation of homogeneous monolayer coverage is able to decrease and even cut off contacts of target elements with a concomitant matrix component so as to eliminate the matrix effect. ${ }^{37-42}$

The two phenomena occurring during the spontaneous dispersion process gave us an inspiration for problems demanding prompt solution in analytical chemistry: matrix interference and solid dilution. Until now, as a novel research field, the spontaneous dispersion theory has not been reported to directly apply to antiinterference and solid dilution. In this work, plastics and rice were chosen as model samples to investigate the solid dilution and antimatrix effect. Based on the spontaneous surface dispersion (SSD) principle, if Cd-containing compounds in rice or plastic samples were ashed and then heated to spontaneously disperse on the support surface, it can accomplish physical separation of the $\mathrm{Cd}$ species from concomitant matrices during vaporization. We undertook SSD of $\mathrm{Cd}$ in solid rice and plastic samples using $\alpha$ $\mathrm{Al}_{2} \mathrm{O}_{3}$ powder as the support. After optimization, quantitative elemental dilution of the solid sample and interference-free solid sampling analysis were successfully achieved. With reliability, precision, simplicity, and green chemistry, such an SSD approach opens a novel way of anti-interference in the inorganic element field of analytical chemistry.

\section{EXPERIMENTAL}

Instrumentation. The solid sampling device (Model DCD-200, Beijing Titan Instruments Co. Ltd, Beijing, P. R. China) comprises an ashing furnace, ETV, tungsten coil trap (TCT), and a sample boat of porous carbon (SANYE Carbon Materials Factory, Shandong, P. R. China); the latter has been described in our previous studies. ${ }^{43,44} \mathrm{~A} 10 \% \mathrm{H}_{2}$-in- $\mathrm{Ar}(v / v)$ mixture was chosen as the carrier. The ETV programs for Cd analysis are slightly modified from previously reported conditions. ${ }^{4,5}$ The AFS (Model AFS-8230, Beijing Titan Instruments, Beijing, P.R. China) was equipped with a $228.8 \mathrm{~nm}$ Cd-boosted hollow cathode lamp (HCL, 
Beijing Research Institute of Nonferrous Metals, Beijing, P.R. China). The parameters are also listed in detail in Table S1. A vacuum/atmosphere tubular resistance furnace (Model LG0612K, Tianjin Laboratory Instrument, Tianjin, P.R. China), a box-type resistance furnace (Model SX2-10-12, Tianjin Zhonghuan Furnace, Tianjin, P.R. China) and a homogenization workstation (Model SiO-6512, Beijing Ability Technique, Beijing, P.R. China) were used in the SSD process of sample preparation. Secondary ion mass spectrometry (SIMS), X-ray diffraction (XRD), and X-ray photoelectron spectroscopy (XPS) were employed for studying the mechanism. SIMS (TOF-SIMS 5-100, ION-TOF GmbH, Münster, Germany) was used to measure the $\mathrm{Cd}$ concentration on the $\alpha-\mathrm{Al}_{2} \mathrm{O}_{3}$ support surface; all of the parameters are listed in Table S2. The XRD (D8 Advance, Bruker Optics, Germany) was used to observe the crystal phase changes after high-temperature treatment; XPS (ESCALAB 250Xi, ThermoFisher, USA) was used to measure the bonding energy of $\mathrm{Cd}$ dispersed on the $\alpha$ $\mathrm{Al}_{2} \mathrm{O}_{3}$ support surface; selection of its excitation source was dependent on the Al Kay (2 keV) X-ray radiation. The ICP-MS (Model EXPEC-7000, Focused Photonics Inc., Hangzhou, P. R. China) was employed for the method verification. The detailed operating parameters of the ICP-MS were as follows: incident RF power, $1600 \mathrm{~W}$; cooling argon gas flow rate, $13.0 \mathrm{~L} / \mathrm{min}$; nebulizer argon gas, $0.85 \mathrm{~L} / \mathrm{min}$; auxiliary argon gas, $0.80 \mathrm{~L} / \mathrm{min}$; the sampling depth was $5 \mathrm{~mm}$ with an inside diameter of $0.02 \mathrm{~mm}$, and the peristaltic pump rate was $30 \mathrm{rpm}$. As for monitoring the isotope, ${ }^{114} \mathrm{Cd}$ was selected using standard mode.

Materials and Reagents. Stock standards of $\mathrm{Cd}, \mathrm{Ca}, \mathrm{Na}, \mathrm{Al}, \mathrm{As}$, $\mathrm{Cr}$, and $\mathrm{Pb}(1000 \mathrm{mg} / \mathrm{L}), \mathrm{Fe}, \mathrm{Cu}, \mathrm{Mn}$, and $\mathrm{Mg}(100 \mathrm{mg} / \mathrm{L})$ and rice, plastic CRMs (GBW100360, GBW100351, GBW100357, GBW10043, GBW10044, GBW08404, GBW08405, and GBW08406) were purchased from the National Research Center for Certified Reference Materials of China (Beijing, P. R. China). The working standard solutions were obtained by stepwise dilution of the stock standards with deionized water, which was prepared using the Milli-Q integral water purification system (Millipore Corporation, USA). All chemicals were of reagent grade and purchased from Sinopharm Chemical Reagent (Beijing,
P. R. China), unless otherwise stated. Powdered $\alpha-\mathrm{Al}_{2} \mathrm{O}_{3}$ was used as supports for SSD. ${ }^{45}$ Before use, the powdered $\alpha-\mathrm{Al}_{2} \mathrm{O}_{3}$ was heated at $1000{ }^{\circ} \mathrm{C}$ for $10 \mathrm{~h}$ in a vacuum/atmosphere tubular resistance furnace in a $10 \% \mathrm{H}_{2}$-in- $\mathrm{Ar}$ atmosphere to vaporize possible $\mathrm{Cd}$ residue in order to eliminate background interference. Zirconia balls (ø $3 \mathrm{~mm}$, Saint-Gobain, Handan, P. R. China) were used to mix the $\alpha-\mathrm{Al}_{2} \mathrm{O}_{3}$ powder with powdered rice or plastic samples using ethanol medium; if necessary, the $\mathrm{Cd}$ standards were diluted in ethanol. Rice samples were purchased from a local supermarket and milled into powder to pass through a 100-mesh sieve.

Sample preparation for SSD using Cd solution. To facilitate $\mathrm{Cd}$ dispersion on the $\alpha-\mathrm{Al}_{2} \mathrm{O}_{3}$ supports, a stepwise premixing process was performed as follows: (1) $\mathrm{Cd}$ standard solution was introduced into a centrifuging tube with $\sim 18 \mathrm{~g} \alpha-\mathrm{Al}_{2} \mathrm{O}_{3}$; (2) $15 \mathrm{~mL}$ alcohol and several zirconia balls were added, then agitated at 1200 cycles per minute (cpm) for 3 min using a homogenization workstation; (3) the content was dried at $100{ }^{\circ} \mathrm{C}$ for $30 \mathrm{~min}$; and finally, (4) the mixture was heated at $700{ }^{\circ} \mathrm{C}$ in the crucible in the box-type resistance furnace for SSD study.

Rice sample preparation and analytical procedures. The schematic diagram of the SSD-based sample preparation and $\mathrm{Cd}$ analysis is shown in Fig. 1. The step-by-step procedures were: (1) $100 \mathrm{mg}$ rice sample and $0.5 \mathrm{~g} \alpha-\mathrm{Al}_{2} \mathrm{O}_{3}$ supports were mixed with $1.5 \mathrm{~mL}$ of alcohol and several zirconia balls, then agitated in advance; (2) the sample was then heated at high temperature and the dispersed sample was introduced into the ETV to vaporize $\mathrm{Cd}$ in the homogenization workstation, vide supra; (3) the sample was pre-ashed at $300{ }^{\circ} \mathrm{C}$ for $30 \mathrm{~min}$ to remove the water and organics under an $\mathrm{Ar} / \mathrm{H}_{2}$ atmosphere; the $\mathrm{Cd}$ vapor was subsequently to evenly disperse $\mathrm{Cd}$ on the $\alpha-\mathrm{Al}_{2} \mathrm{O}_{3}$ surface; (4) a $5 \mathrm{mg}$ dispersed sample was introduced int the ETV to vaporize $\mathrm{Cd}$ under an $\mathrm{Ar} / \mathrm{H} 2$ atmosphere; the Cd vapor was subsequently trapped by a TCT in the trapping chamber; (5) the TCT was heated to release Cd at an $\mathrm{Ar} / \mathrm{H}_{2}$ flow of $600 \mathrm{~mL} / \mathrm{min}$ for AFS measurement.

Plastic sample preparation and analytical procedures. The procedures were: (1) 100 mg plastic sample and $\sim 18 \mathrm{~g} \alpha-\mathrm{Al}_{2} \mathrm{O}_{3}$

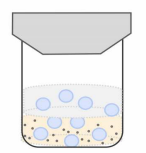

(b) Vibrating

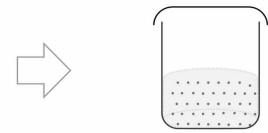

(c) Heating for SPD

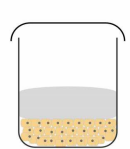

(a) Pre-carbonization

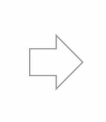

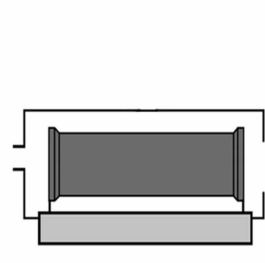

(e) Vaporization

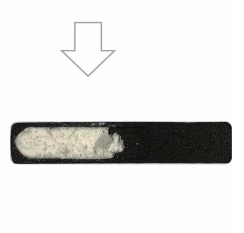

(d) Soild sampling

(f) Trapping and releasing

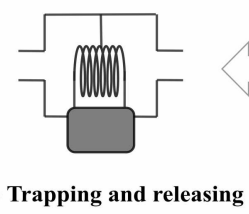

Fig. 1 The schematic diagram of solid sampling analysis of $\mathrm{Cd}$ in real sample coupled based on spontaneous dispersion. 
supports were mixed, placed into a quartz crucible and heated at $300{ }^{\circ} \mathrm{C}$ for $1 \mathrm{~h}$ to carbonize the organic substances; (2) the ash was mixed with $15 \mathrm{~mL}$ of alcohol and several added zirconia balls, then agitated, vide supra; (3) the content was equally divided into several aliquots, one of which was then heated at high temperature to evenly disperse $\mathrm{Cd}$ on the $\alpha-\mathrm{Al}_{2} \mathrm{O}_{3}$ surface; (4) the testing procedure was performed, vide supra.

\section{RESULTS AND DISCUSSION}

Premixing for Cd SSD. For SSD, in addition to the properties and structures of the solid compound-support pair, the temperature is the critical factor to achieve spontaneous dispersion. At a so-called critical dispersion temperature (Tc, near Tammann temperature), the entropy effect becomes the governing factor, and the surface diffusion velocity approaches that in the solution media. Thus, the solid compounds disperse on the support surface at an appreciable rate. However, an ultrahigh temperature could lead to solidsupport inter-reaction. According to the spontaneous dispersion theory, ${ }^{37,38}$ the substances that disperse spontaneously as described are almost ionic or molecular crystalline compounds, while in ashed rice or plastic samples, the elements of interest usually exist as metallic oxides or salts dispersed on the support surface. As target elements disperse on a support surface into a homogeneous state, this virtually causes multi-fold dilution due to the large specific surface area of the supports.

To verify the occurrence of SSD, Cd standards were used on the $\alpha-\mathrm{Al}_{2} \mathrm{O}_{3}$ surface prior to testing a real sample. First, manual grinding was carried out to mix the Cd standard with the $\alpha-\mathrm{Al}_{2} \mathrm{O}_{3}$ supports. Unfortunately, grinding for more than $1 \mathrm{~h}$ failed to sufficiently homogenize $\mathrm{Cd}$ as was evidenced by $>45 \% \mathrm{RSD}$ (each sampling took place from a random point in the mixed sample). Furthermore, the RSD of a ground sample was not significantly reduced after heating at $700{ }^{\circ} \mathrm{C}$ for $4 \mathrm{~h}$. In theory, the long-range move of an analyte in liquid medium mainly relies on convection during homogenization; while short-range dispersion depends on analyte diffusion, which can be quickly reached via SSD. However, lack of analyte convection in a solid render it difficult to move an analyte as fast over a long range than over a short range. Thus, it is impossible to disperse $\mathrm{Cd}$ within several hours by relying only on thermal drive. In order to help an analyte to move to long range quickly, premixing must be employed to assist $\mathrm{Cd}$ homogenization on the support before SSD. An alternative is to dip the $\alpha-\mathrm{Al}_{2} \mathrm{O}_{3}$ supports in $15 \mathrm{~mL}$ ethanol containing $\mathrm{Cd}$ and with several zirconia balls, place them into a centrifuging tube, and then agitate the mixture using a homogenization workstation at $1200 \mathrm{cpm}$. The ethanol medium not only promotes the convection in long range, but also volatilizes rapidly after agitation. After optimization, the RSDs were reduced to $<15 \%$ with 3 min agitation, demonstrating better dispersion of
$\mathrm{Cd}$ on the $\alpha-\mathrm{Al}_{2} \mathrm{O}_{3}$ support. As a result, agitation was included for the study of thermal treatment based on SSD, vide infra.

Critical dispersion temperature for Cd SSD. Temperature is of utmost importance to SSD; Tc plays a crucial role in initiation of $\mathrm{SSD}$. To investigate SSD dependence on temperature, $3.6 \mu \mathrm{g} \mathrm{Cd}$ (0.36 mL standard solution) was mixed with $18 \mathrm{~g} \alpha-\mathrm{Al}_{2} \mathrm{O}_{3}$ supports as mentioned, and one aliquot was heated for $4 \mathrm{~h}$ (in excess to ensure a sufficient dispersion) under varied temperatures $\left(300{ }^{\circ} \mathrm{C}\right.$ to $\left.900{ }^{\circ} \mathrm{C}\right)$. Then, $5 \mathrm{mg}$ dispersed sample was measured for $\mathrm{Cd}$ by random sampling. As shown in Fig. 2, the average $\mathrm{Cd}$ intensities remained constant at $<700{ }^{\circ} \mathrm{C}$ and significantly decreased at $>800{ }^{\circ} \mathrm{C}$. On the other hand, the RSD without heat treatment exceeded $14 \%$; heating below $400{ }^{\circ} \mathrm{C}$ did not improve the RSD either. On the other hand, heating up to $600{ }^{\circ} \mathrm{C}$ significantly reduced the RSD to $<5 \%$, indicating that SSD took place between 600 and $700{ }^{\circ} \mathrm{C}$. Therefore, Tc should be in this range. At $>800{ }^{\circ} \mathrm{C}$, the $\mathrm{RSD}$ rose likely due to the worsened heterogeneity caused by uneven $\mathrm{Cd}$ loss, and followed the trend of declining Cd concentration (Fig. 2). Reduced RSD implied that SSD occurred to $\mathrm{Cd}$ on the $\alpha-\mathrm{Al}_{2} \mathrm{O}_{3}$ support. In addition, Fig. S1 shows that $30 \mathrm{~min}$ heating at $700{ }^{\circ} \mathrm{C}$ can reach the optimal $\mathrm{Cd}$ diffusion with $\sim 5 \%$ RSD. Nevertheless, direct evidence employing surface analysis is still needed.

Evidence of SSD occurrence to $\mathrm{Cd}$ on $\alpha$ - $\mathrm{Al}_{2} \mathrm{O}_{3}$ support. In previous studies on heterogeneous catalysis, XPS and XRD were utilized to prove SSD occurrence based on the binding energy and crystal phase of the support surface. ${ }^{37,46,47}$ We thereby applied XPS and XRD to obtain evidence of SSD occurrence to Cd on the $\alpha$ $\mathrm{Al}_{2} \mathrm{O}_{3}$ support. Before heating, the $\alpha-\mathrm{Al}_{2} \mathrm{O}_{3}$ supports were dipped into $15 \mathrm{~mL}$ ethanol containing $\mathrm{Cd}$ and adding several zirconia balls; the mixture was dried following the above-mentioned protocol to attain samples spiked with $\mathrm{Cd}$ at $0,0.5,1$, and $10 \mu \mathrm{g} / \mathrm{g}$. Then, the prepared mixtures were heated for SSD under $600^{\circ} \mathrm{C}$ for $30 \mathrm{~min}$. XRD and XPS measurements revealed no $\mathrm{Cd}$, probably due to ultralow surface $\mathrm{Cd}$ concentration. In the previous XRD and XPS

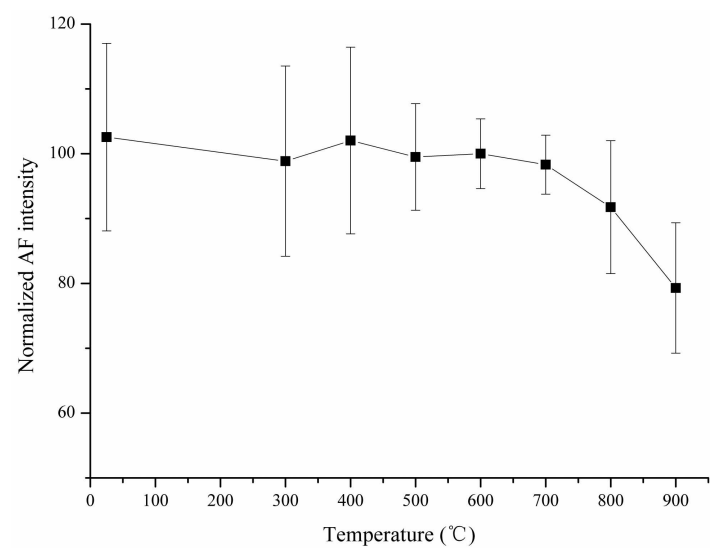

Fig. 2 Effect of temperatures on $\mathrm{Cd}$ dispersion on $\alpha-\mathrm{Al}_{2} \mathrm{O}_{3}$ supports using standard solution $(n=3)$. AF intensity of $\mathrm{Cd}$ at $600^{\circ} \mathrm{C}$ is set as 100 , and others are normalized. 
Table 1. Cd/Al on $\alpha-\mathrm{Al}_{2} \mathrm{O}_{3}$ Support's Surface Measured by SIMS

\begin{tabular}{lc}
\hline Sample preparation & $\mathbf{C d} / \mathbf{A l}^{\mathrm{a}}$ \\
\hline Before heating & 0.5 \\
After heating & 1.2 \\
\hline
\end{tabular}

${ }^{a} \mathrm{Cd} / \mathrm{Al}$ means the ratio of $\mathrm{Cd}$ signal to that of Al by SIMS

studies, SSD occurred on supports at high concentrations; ; $;$,46,47 however, such high elemental concentrations were not suited for real sample analysis. Thus, we employed SIMS to measure $\mathrm{Cd}$ on the $\alpha-\mathrm{Al}_{2} \mathrm{O}_{3}$ support using a prepared sample spiked at $5 \mu \mathrm{g} / \mathrm{g} \mathrm{Cd}$. Considering the mutual incomparability of $\mathrm{Cd}$ and Al SIMS signals, peak area ratio $\mathrm{Cd} / \mathrm{Al}$ was employed to evaluate $\mathrm{Cd}$ dispersion, which was proportional to the $\mathrm{Cd}$ concentration on the $\alpha-\mathrm{Al}_{2} \mathrm{O}_{3}$ surface. From Table 1 , the $\mathrm{Cd} / \mathrm{Al}$ value after $600{ }^{\circ} \mathrm{C}$ heating was significantly higher than that without thermal treatment, indicating an increase in $\mathrm{Cd}$ concentration on the shallow $\alpha-\mathrm{Al}_{2} \mathrm{O}_{3}$ support surface.

Prior to heating, $\mathrm{Cd}$ exists probably on the $\alpha-\mathrm{Al}_{2} \mathrm{O}_{3}$ support as multi-layers or aggregates. Because SIMS only detects atoms on a very shallow surface with an ion beam, the average signal from the projected area covering $\mathrm{Cd}$ aggregates and the blank surface (no $\mathrm{Cd}$ occupation) should be lower than that from an even $\mathrm{Cd}$ monolayer. So, the increase of $\mathrm{Cd} / \mathrm{Al}$ ratio is probably due to the formation of a $\mathrm{Cd}$ monolayer on the $\alpha-\mathrm{Al}_{2} \mathrm{O}_{3}$ support. This can be considered as a direct evidence of Cd SSD that occurred only at an adequately high temperature. Furthermore, a reduced RSD aligned with the SIMS results supported SSD occurrence. Considering the relatively low $\mathrm{Cd}$ concentration dispersed on the support in real matrices, SIMS could not provide adequate signals. As a result, for real samples, a decreased RSD was selected as the proper evidence of SSD in the following experiment.

Solid dilution study. The above results showed a promising quantitative solid dilution via SSD. Taking real plastic samples (CRMs) as model analyte, solid particles made of organic polymeric materials are hardly mixable with the $\alpha-\mathrm{Al}_{2} \mathrm{O}_{3}$ supports As usual, ashing-acid digestion especially for plastics is timeconsuming and requires toxic reagents prior to atomic spectrometric analysis. Moreover, $\mathrm{Cd}$ in plastics is apt to be lost at high temperatures during a direct ashing process due to rich organic substances. Thus, plastic sample analysis prefers solid dilution to replace acid digestion.

To obtain fine particulates for homogenization, organic components must be decomposed or completely removed prior to SSD. Therefore, it is indispensable to ash plastic samples to convert $\mathrm{Cd}$ to oxides or salts. Thereby, a mixture of plastic sample and $\alpha-\mathrm{Al}_{2} \mathrm{O}_{3}$ support was ashed in a quartz crucible. After optimization, $300{ }^{\circ} \mathrm{C}$ heating in a box-type resistance furnace for $1 \mathrm{~h}$ was able to achieve preliminary carbonization without $\mathrm{Cd}$ loss. Similar to the case using standard solutions, high temperature alone failed to disperse $\mathrm{Cd}$ on the $\alpha-\mathrm{Al}_{2} \mathrm{O}_{3}$ support for real samples taking long-range SSD into account. The ash together with several zirconia balls was agitated at $1000 \mathrm{cpm}$ for $3 \mathrm{~min}$ in $15 \mathrm{~mL}$ ethanol.
Based on the above-mentioned preparation protocol, the combination of ashing and agitation reduced the RSD to $27 \%$ before plastic SSD heating.

To verify SSD occurrence to $\mathrm{Cd}$ in real samples, $96.6 \mathrm{mg}$ of CRM GBW08405 Plastic was mixed with $17.836 \mathrm{~g}$ of $\alpha-\mathrm{Al}_{2} \mathrm{O}_{3}$ based on the above-mentioned protocol. One aliquot of the mixed sample was heated for $4 \mathrm{~h}$ at varied temperatures $\left(300{ }^{\circ} \mathrm{C}\right.$ to $800^{\circ} \mathrm{C}$ ); then, $\sim 3 \mathrm{mg}$ sample was measured for $\mathrm{Cd}$ presence. As shown in Fig. $3 \mathrm{a}, 600^{\circ} \mathrm{C}$ is the turning point of $\mathrm{Cd}$ content decline. The RSD of $27 \%$ was reached without heating, but reduced to $8 \%$ at $600{ }^{\circ} \mathrm{C}$. It was also indicative of effective dispersion on the $\alpha$ $\mathrm{Al}_{2} \mathrm{O}_{3}$ support. On the other hand, worse RSD and a declining value were observed at $>700^{\circ} \mathrm{C}$, which could be attributed to carbides burning at high temperature. Here, carbides burning off caused a significant rise in temperature and accelerated Cd loss. ${ }^{48}$ As a result, $600{ }^{\circ} \mathrm{C}$ was set for the following dynamic study for plastic sample with SSD.

Based on the above study, $\mathrm{Cd}$ from real plastic samples underwent SSD with $\alpha-\mathrm{Al}_{2} \mathrm{O}_{3}$ support well below Tc at $600{ }^{\circ} \mathrm{C}$. To investigate the dynamic conditions of Cd during SSD, as shown in Fig. 3b, $103.4 \mathrm{mg}$ of CRM GBW08405 Plastic was mixed with $19.085 \mathrm{~g} \alpha-\mathrm{Al}_{2} \mathrm{O}_{3}$ and set at $600{ }^{\circ} \mathrm{C}$, based on the protocols mentioned above; one aliquot was heated at different time durations (10 to $60 \mathrm{~min}$ ). Next, $\sim 3 \mathrm{mg}$ dispersed sample was measured for $\mathrm{Cd}$ presence. The average $\mathrm{Cd}$ content $v$ s. heating time from 20 to $60 \mathrm{~min}$ revealed no significant difference, indicating no loss of $\mathrm{Cd}$ under $600^{\circ} \mathrm{C}$. The RSD was $23 \%$ without heating, but reduced to a minimum of $9 \%$ after 30 min heating with a plateau of $\mathrm{Cd}$ presence. Homogeneous dispersion of $\mathrm{Cd}$ from the real plastic sample onto the $\alpha-\mathrm{Al}_{2} \mathrm{O}_{3}$ support was fulfilled using $600{ }^{\circ} \mathrm{C}$ heating for more than $30 \mathrm{~min}$. Thus, $600{ }^{\circ} \mathrm{C}$ and 30 min were set as the optimal SSD conditions for solid dilution of plastic samples resulting in $\sim 180$-fold favorable homogeneity.

Thermodynamic conditions and dynamic conditions of SSD for $\mathrm{Cd}$ in rice sample. To overcome long-range $\mathrm{SSD}$, the rice powder and $\alpha-\mathrm{Al}_{2} \mathrm{O}_{3}$ support were mixed, zirconia balls added, then agitated in ethanol medium and homogenization at $1200 \mathrm{cpm}$ for $3 \mathrm{~min}$. After optimization, $300{ }^{\circ} \mathrm{C}$ carbonization in a box-type resistance furnace for $30 \mathrm{~min}$ achieved complete carbonization without $\mathrm{Cd}$ loss. Based on the above-mentioned preparation protocol, the combination of agitation and ashing reduced the RSD to $<20 \%$ before SSD heating of rice. To verify SSD of Cd in real samples, $100 \mathrm{mg}$ of CRM GBW10045 Rice was mixed with $0.5 \mathrm{~g}$ $\alpha-\mathrm{Al}_{2} \mathrm{O}_{3}$ and the above-mentioned protocol followed. One aliquot of the mixed sample was heated for $4 \mathrm{~h}$ at various temperatures $\left(200{ }^{\circ} \mathrm{C}\right.$ to $\left.700{ }^{\circ} \mathrm{C}\right)$; then $\sim 5 \mathrm{mg}$ sample was measured for $\mathrm{Cd}$. As shown in Fig. $3 \mathrm{c}, 400{ }^{\circ} \mathrm{C}$ is the turning point at which the $\mathrm{Cd}$ concentration plateaued. The RSD reached $18 \%$ without heating, but reduced to $1.7 \%$ at $700{ }^{\circ} \mathrm{C}$. It was also indicative of effective dispersion on the $\alpha-\mathrm{Al}_{2} \mathrm{O}_{3}$ support. As a result, $700{ }^{\circ} \mathrm{C}$ was set for the following dynamic study on rice analysis using SSD. 

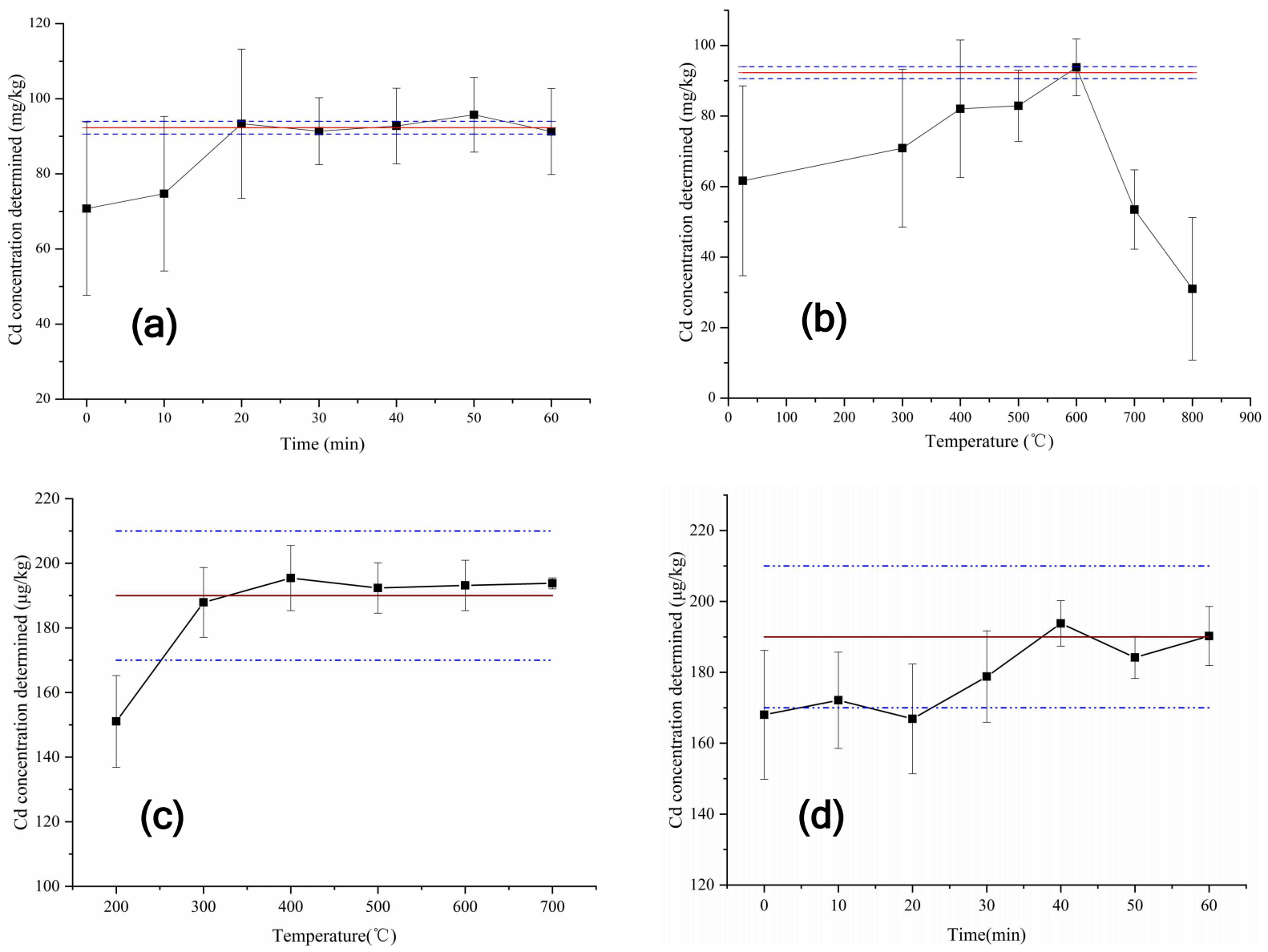

Fig. 3 a) Thermodynamic conditions of $\mathrm{Cd}$ dispersion on $\alpha-\mathrm{Al}_{2} \mathrm{O}_{3}$ supports using real plastic samples $(\mathrm{n}=3)$; ) Dynamic conditions of $\mathrm{Cd}$ dispersion on $\alpha$ $\mathrm{Al}_{2} \mathrm{O}_{3}$ supports using real plastic samples $(n=3)$; c) Thermodynamic conditions of $\mathrm{Cd}$ dispersion on $\alpha-\mathrm{Al}_{2} \mathrm{O}_{3}$ supports using real rice samples $(n=3)$; $d$ ) Dynamic conditions of $\mathrm{Cd}$ dispersion on $\alpha-\mathrm{Al}_{2} \mathrm{O}_{3}$ sup-ports using real rice samples $(\mathrm{n}=3)$. The solid red line and the solid brown line are the certified value of Cd in plastic sample (CRM, GBW08405) and rice sample (CRM, GBW10045), respectively. The blue broken lines are the lower and upper limits in the uncertainty ranges, respectively.

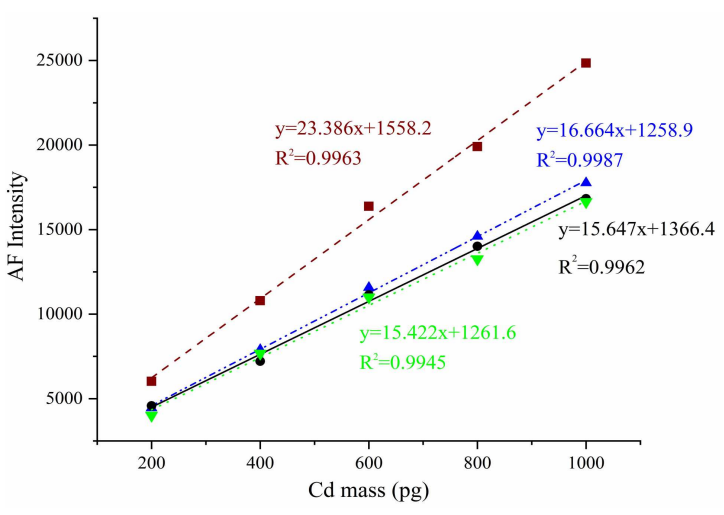

Fig. 4 Effect of different calibration strategies on matrix interference. Blank points and line show standard curve; green shows standard $+\alpha-\mathrm{Al}_{2} \mathrm{O}_{3}$; red shows rice $\mathrm{CRM}$; blue shows $\mathrm{CRM}+\alpha-\mathrm{Al}_{2} \mathrm{O}_{3}$.

Based on the above study on $\mathrm{Al}_{2} \mathrm{O}_{3}$, Cd from a real rice sample underwent SSD on $\alpha-\mathrm{Al}_{2} \mathrm{O}_{3}$ support well below Tc at $700{ }^{\circ} \mathrm{C}$. To investigate the dynamic conditions of Cd during $\mathrm{SSD}$, as shown in Fig. 3d, $100 \mathrm{mg}$ of CRM GBW10045 Rice was mixed with $0.5 \mathrm{~g}$ $\alpha-\mathrm{Al}_{2} \mathrm{O}_{3}$ at $700{ }^{\circ} \mathrm{C}$, based on the protocols mentioned above; one aliquot was heated using different time durations (10 to $60 \mathrm{~min}$ ). Next, $5 \mathrm{mg}$ dispersed sample was measured for $\mathrm{Cd}$. The average $\mathrm{Cd}$ content $v$ s. heating time revealed no obvious difference from 0 to $20 \mathrm{~min}$, indicating no loss of $\mathrm{Cd}$ at $600{ }^{\circ} \mathrm{C}$. The RSD was $21 \%$ without heating; but reduced to a minimum of $6 \%$ using $40 \mathrm{~min}$ heating, and then plateaued. Homogeneous dispersion of $\mathrm{Cd}$ from a real rice sample onto the $\alpha-\mathrm{Al}_{2} \mathrm{O}_{3}$ support was completed by heating at $700{ }^{\circ} \mathrm{C}$ for more than $40 \mathrm{~min}$. However, the main aim of this investigation is not for solid dilution of a model rice sample, but rather than for its anti-interference effect. Thus, $700{ }^{\circ} \mathrm{C}$ and 40 min were considered as the optimal SSD conditions to investigate the matrix interference.

Interference study. Due to complicated concomitant interference during vaporization, the previous solid sampling method cannot obtain interference-free measurement of $\mathrm{Cd}$ in the rice sample. In practice, matrix matching calibration is time-consuming, inconvenient and frequently lacks availability of proper CRMs. To investigate the anti-interference effect, different calibration curves are shown in Fig. 4, including standard solution, standards $+\alpha$ $\mathrm{Al}_{2} \mathrm{O}_{3}$ (SSD), CRM GBW10045 Rice, and rice CRM $+\alpha-\mathrm{Al}_{2} \mathrm{O}_{3}$ 
Table 2. Measured Cd Presence and Recoveries in Real Samples $(\mathrm{ng} / \mathrm{g}, \mathrm{n}=3)$

\begin{tabular}{|c|c|c|c|c|}
\hline Samples & Certified or ICP-MS & Previous method $^{\mathrm{a}}$ & Proposed method ${ }^{\mathrm{b}}$ & Corrective ratio (\%) \\
\hline Rice 1 & $11.9 \pm 0.1$ & $13.3 \pm 2.7$ & $10.9 \pm 1.9$ & -18 \\
\hline Rice 2 & $6.0 \pm 0.7$ & $7.0 \pm 0.6$ & $5.9 \pm 0.1$ & -15.7 \\
\hline Rice (GBW(E)100360) & $220 \pm 20$ & $241 \pm 14$ & $214 \pm 17$ & -11.2 \\
\hline Rice $(\mathrm{GBW}(\mathrm{E}) \mathbf{1 0 0 3 5 1 )}$ & $420 \pm 20$ & $448 \pm 4$ & $417 \pm 10$ & -6.9 \\
\hline Rice $(\mathrm{GBW}(\mathrm{E}) 100357)$ & $216 \pm 16$ & $233 \pm 6$ & $216 \pm 12$ & -7.3 \\
\hline Rice (GBW10043) & $12 \pm 3$ & $15 \pm 1$ & $11 \pm 1.6$ & -26.7 \\
\hline Rice (GBW10044) & $18 \pm 2$ & $20.9 \pm 0.5$ & $17.5 \pm 1.6$ & -16.3 \\
\hline Plastic (GBW08404) & $9.26 \pm 0.22$ & - & $9.1 \pm 0.3$ & - \\
\hline Plastic (GBW08406) & $36.2 \pm 0.7$ & 一 & $37.0 \pm 2.0$ & - \\
\hline Plastic (GBW08405) & $92.3 \pm 1.7$ & - & $91.2 \pm 4.5$ & - \\
\hline
\end{tabular}

${ }^{a}$ Measured value by ETV-TCT-AFS without SSD; ${ }^{b}$ Measured value by the proposed SSD method; ${ }^{\mathrm{c}}$ Indicates (b-a)/a×100\% according to the mean value from $\mathrm{a}$ and $\mathrm{b}$, vide supra.

Table 3. Effect of Possible Inorganic Elements

\begin{tabular}{lcc}
\hline Elements & Added $(\mathbf{m g} / \mathbf{L})$ & Recoveries $(\%)$ \\
\hline $\mathbf{C a}$ & 100 & 105 \\
$\mathbf{F e}$ & 10 & 102 \\
$\mathbf{C u}$ & 10 & 88 \\
$\mathbf{M n}$ & 10 & 91 \\
$\mathbf{N a}$ & 1000 & 97 \\
$\mathbf{M g}$ & 200 & 99 \\
$\mathbf{A l}$ & 200 & 103 \\
$\mathbf{A s}$ & 10 & 115 \\
$\mathbf{C r}$ & 10 & 110 \\
$\mathbf{P b}$ & 10 & 95 \\
\hline
\end{tabular}

(SSD). First, the calibration curves of standard and standard $+\alpha$ $\mathrm{Al}_{2} \mathrm{O}_{3}$ are almost overlapped, indicating no extra influence from the $\alpha-\mathrm{Al}_{2} \mathrm{O}_{3}$ as support. However, the matrix matching calibration curve, namely the rice CRM curve, obviously deviates from the standard and standard $+\alpha-\mathrm{Al}_{2} \mathrm{O}_{3}$ calibrations. Here, matrix interference proved to exist again. So, it would lead to a positivegoing deviation, yielding higher results than the true values as listed in Table 2. On the other hand, the rice $\mathrm{CRM}+\alpha-\mathrm{Al}_{2} \mathrm{O}_{3}$ curve, namely SSD treatment, is very close to the standard and standard $+\alpha-\mathrm{Al}_{2} \mathrm{O}_{3}$ calibrations, demonstrating no significant interference. The measured $\mathrm{Cd}$ by the solid sampling analysis method using the standard solution strategy is within the certified values of the CRMs. Thus, the matrix interference can be corrected via the SSD protocol, where the corrective ratios range from $-6.9 \%$ to $-26.7 \%$ as compared without SSD. In other words, the solid dilution via SSD results in the complete elimination of matrix interference in the model solid sample used for this study.

To investigate a potential interference by other elements to $\mathrm{Cd}$ determination based on the proposed method, $\mathrm{Ca}, \mathrm{Fe}, \mathrm{Cu}, \mathrm{Mn}, \mathrm{Na}$, $\mathrm{Mg}, \mathrm{Al}, \mathrm{As}, \mathrm{Cr}$, and $\mathrm{Pb}$ with $10 \mathrm{mg} / \mathrm{L}$ to $1000 \mathrm{mg} / \mathrm{L}$ levels were added to real samples (GBW10045) and then were dried at $40{ }^{\circ} \mathrm{C}$ using a drying oven. In Table 3, 88-115\% Cd recoveries indicate absence of interference, which can be attributed in part to the intrinsic advantage of SSD.

\section{CONCLUSIONS}

A novel solid dilution method for anti-matrix interference was first rendered and verified based on the SSD principle originally applied to the synthesis of heterogeneous catalysts. After preashing and assistant agitation, $\mathrm{Cd}$ in the solid sample can be efficiently and quickly diluted via SSD on $\alpha-\mathrm{Al}_{2} \mathrm{O}_{3}$ support at critical temperature $\left(600-700^{\circ} \mathrm{C}\right)$ for $30-40 \mathrm{~min}$, in which $>180$ fold dilution was achieved and the mechanism confirmed and deduced. In essence, the proposed SSD is a solid dilution process on the support's surface near Tammann temperature where homogeneous monolayer coverage forms in order to reduce and even to avoid contact of the target elements with interferents. Thus, the anti-interference effect has been completely verified via matrix interference evaluation for $\mathrm{Cd}$ in the model rice sample. Moreover, the SSD solid dilution process requiring only low cost $\alpha-\mathrm{Al}_{2} \mathrm{O}_{3}$ for SSD and solid sampling analysis after ashing, offers the advantages of reliability, precision, simplicity, and green chemistry. The SSD theory is revolutionary for the dilution of solids and thoroughly different from the previous anti-interference technical routines. Though the mechanism of this SSD approach needs to be further explored for more elements, it potentially will lead to a novel theory for anti-interference research.

\section{ASSOCIATED CONTENT}

Please contact the corresponding author for the Supporting Information (Table S1 and Table S2).

\section{AUTHOR INFORMATION}

\section{Corresponding Author}

*X. F. Mao

E-mail address:mxf08@163.com; maoxuefei@caas.cn

*J. X. Liu

E-mail address: 1jx2117@gmail.com

Notes

B. Wang and T. P. Liu contributed equally to this work. 


\section{ACKNOWLEDGMENTS}

This investigation was funded by the National Natural Science Fund of China (No. 31571924), the National Key Research and Development Program of China (No. 2017YFD0801203) and the Central Public-interest Scientific Institution Basal Research Fund (No. Y2019XK05/ 1610072019001). In addition, sincerest thanks to the support of the Agricultural Science and Technology Innovation Program of CAAS, presided over by Yongzhong Qian and Jing Wang (No. CAAS-XTCX2019024), respectively.

\section{REFERENCES}

1. B. V. L'vov, J. Anal. Chem., 2005, 60, 382-392.

2. T. Panyabut, N. Sirirat and A. Siripinyanond, Anal. Chim. Acta, 2018, 1000, 75-84.

3. H. Cui, W. Guo, L. L. Jin, Q. H. Guo and S. H. Hu, Anal. Methods, 2017, 9, 1307-1312.

4. B. Wang, L. Feng, X. F Mao, J. X. Liu, C. C. Yu, L. Ding, S. Q. Li, C. M. Zheng, Y. Z. Qian, J. Anal. At. Spectrom., 2016, 33, 12091216.

5. Y. Zhang, X. F. Mao, M. Wang, C. L. Gao, Y. H. Qi, Y. .Z Qian, X. Y. Tang and J. Zhou, Anal. Lett., 2015, 48, 2908-2920.

6. N. S. Medvedeva, O. V. Lundovskayaa and A. I. Saprykin, Microchem. J., 2019, 145, 751-755.

7. F. Borno, S. Richter, D. Deiting, N. Jakubowski, U. Panne, J. Anal. At. Spectrom., 2015, 30, 1064-1071.

8. Y. Zhang, X. F. Mao, J. X. Liu, M. Wang, Y. Z. Qian, C. L. Gao and Y. H. Qi, Spectrochim. Acta. B., 2016, 118, 119-126.

9. X. F. Mao, Y. Zhang, J. X. Liu, M. Wang, Y. Z. Qian, Z. Y. Zhang, Y. H. Qi and C. L. Gao, RSC Adv., 2016, 6, 48699-48707.

10. S. Z. Chen, S. P. Zhu and D. B. Lu, At. Spectrom., 2018, 39, 55-61.

11. Y. X. Ye, W. Dong, Y. Luo, T. Fan., X. Y. Xiong, L. Sun and X. X. Hu, Sci. Total. Environ., 2020, 711, 134534.

12. Q. Mahmood, M. Hassan, S. H. Ahmad, K. Bhamu, A. Mahmood and S. M. Ramay, J. Phys. Chem. Solids, 2019, 128, 253-290.

13. T. Zangmo and A. Siripinyanond, Anal. Chim. Acta, 2019, 1085, 29-38.

14. Q. F. Lu, F. F. Feng, J. Yu, L. Yin, Y. J. Kang, H. Luo, D. X. Sun and W. Yang, Microchem. J., 2020, 152, 104308.

15. A. I. Barros, D. V. Babos, E. C. Ferreira and J. A. G. Neto, Talanta, 2016, 161, 547-553.

16. L. Husáková, I. Urbanová, M. Šafránková and T. Š́dová, Talanta, 2017, 175, 93-100.

17. R. Araujo, N. Oleszczuk, R. T. Rampazzo, P. A. Costa, M. M. Silva M. G. R. Vale, B. Welz and S. Ferreira, Talanta, 2008, 77, 400-406.

18. P. C. Kruger and P. J. Parsons, Spectrochim. Acta. B., 2007, 62, 288-296.

19. F. G. Lepri, B. Wel, M. B. Dessuy, M. G. R. Vale, D. Bohrer, M. T. C. Vollebregt, M. D. Huang and H. B. Ross, Spectrochim. Acta. B., 2010, 65, 24-32.

20. I. Silva, A. F. Lavorante, A. Paim, M. Silva and J. da Food Chem., 2020, 319, 126435.
21. J. T. P. Barbosa, C. M. M. Santos, V. N. Peralva, E. M. M. Flores, M. Korn, J. A. Nóbrega and M. G. A. Korn, Food Chem., 2015, 175, 212-217.

22. L. Tormen, R. A. Gil, V. L. A. Frescura, L. D. Martinez and A. Curtius, Anal. Chim. Acta, 2012, 717, 21-27.

23. A. Passos, M. Dessuy, F. V. Nakadi, J. B. Andrade and M. G. R. Vale, Talanta, 2019, 204, 206-212.

24. B. M. Soares, R. F. Santos, R. C. Bolzan, E. I. Muller, E. G. Primel and F. A. Duarte, Talanta, 2016, 160, 454-460.

25. N. Sadiq and D. Beauchemin, Anal. Chim. Acta, 2014, 851, 23-29.

26. L. Q. Zhao, T. Ren and R. G. Zhong, Anal. Lett., 2012, 45, $2467-$ 2481.

27. J. Teran-Baamonde, A. Carlosena, R. M. Soto-Ferreiro, J. M. Andrade, A. Cantarero-Roldan and S. Muniategui-Lorenzoa, $J$ Anal. At. Spectrom., 2020, 35, 580-591.

28. P. P. Su, P. Ek and A. Ivaska, Holzforschung, 2012, 66, 833-840.

29. J. Malherbe, F. Claverie, A. Alvarez, B. Fern'andez, R. Pereiro, J. L. Molloy, Anal. Chim. Acta, 2013, 793, 72-78.

30. E. M. Hondrogiannis, E. Ehrlinger, A. Poplaski and M. J. Lisle, J. Agric. Food Chem., 2013, 61, 11332-11337.

31. M. G. R. Vale, N. Oleszczuk and W. N. L. Santos, Appl. Spectrosc. Rev., 2006, 41, 377-400.

32. M. Resano, F. Vanhaecke and M. Loos-Vollebregt, J. Anal. Atom. Spectrom., 2008, 23, 1450-1475.

33. Y. Wang, L. S. Lin, B. S. Zhu, Y. X. Zhu and Y. C. Xie, Appl. Surf. Sci., 2008, 254, 6560-6567.

34. C. J. Tang, J. F. Sun, X. J. Yao, Y. Cao, L. C. Liu, C. Y. Ge, F. Gao and L. Dong, Appl. Catal. B-Environ., 2014, 146, 201-212.

35. I. E. Wachs, Dalton T., 2013, 42, 11762-11769.

36. X. Y. Wang, M. Wang and B. Y. Zhao, Acta Phys-chim Sin., 1998, 14, 869-873.

37. Y. C. Xie and Y. Q. Tang, Adv. Catal., 1990, 37, 1-43.

38. Y. C. Xie, Y. X. Zhu, B. Y. Zhao and Y. Q. Tang, Stud. Surf. Sci. Catal., 1998, 118, 441-449.

39. X. Wang, J. S. Tian, Y. H. Zheng, Y. H. Zheng, X. L. Xu, W. M Liu and X. Z. Fang, Chem. Cat. Chem., 2014, 6, 1604-1611.

40. X. Wang, B. Zhao, D. E. Jiang and Y. C. Xie, Appl. Catal. A., 1999, 188, 201-209.

41. C. Deng, Chem., 2007, 70, 900-907.

42. H. Zhu, M. Shen and Y. Wu, J. Phys. Chem., B., 2005, 109, 1172011726.

43. L. Feng and J. X. Liu, J. Anal. At. Spectrom., 2010, 25, 1072-1078.

44. X. F. Mao, J. X. Liu, Y. Huang, L. Feng, L. H. Zhang, X. Y. Tang, J. Zhou, Y. Z. Qian and M. Wang, J. Agr. Food Chem., 2013, 61, 848-853.

45. B. Zhang, N. Z. Wu, Y. C. Xie and Y. Q. Tang,. Acta Physchim. Sin., 1998, 14, 358-360.

46. L. Li, S. H. Chai, A. Binder, S. Brown, G. M. Veith and S. Dai, Catal. Lett., 2014, 144, 912-919.

47. W. X. Xu, L. C. Wang, R. Wang and X. C. Fu, J. Colloid Interf. Sci., 1996, 179, 350-356.

48. P. Wang, L. Lin, Y. X. Zhu and Y. C. Xie. J. Am. Ceram. Soc., 2007, 90, 402-406 